DESY 10-076

\title{
Tetraquark-based analysis and predictions of the cross sections and distributions for the processes $e^{+} e^{-} \rightarrow \Upsilon(1 S)\left(\pi^{+} \pi^{-}, K^{+} K^{-}, \eta \pi^{0}\right)$ near $\Upsilon(5 S)$
}

\author{
Ahmed Ali.* Christian Hambrock $\theta$ and Satoshi Mishimat \\ Deutsches Elektronen-Synchrotron DESY, D-22607 Hamburg, Germany
}

(Dated: March 10, 2011)

\begin{abstract}
We calculate the cross sections and final state distributions for the processes $e^{+} e^{-} \rightarrow$ $\Upsilon(1 S)\left(\pi^{+} \pi^{-}, K^{+} K^{-}, \eta \pi^{0}\right)$ near the $\Upsilon(5 S)$ resonance based on the tetraquark hypothesis. This framework is used to analyse the data on the $\Upsilon(1 S) \pi^{+} \pi^{-}$and $\Upsilon(1 S) K^{+} K^{-}$final states [K. F. Chen et al. (Belle Collaboration), Phys. Rev. Lett. 100, 112001 (2008); I. Adachi et al. (Belle Collaboration), arXiv:0808.2445, yielding good fits. Dimeson invariant mass spectra in these processes are shown to be dominated by the corresponding light scalar and tensor states. The resulting correlations among the cross sections are worked out. We also predict $\sigma\left(e^{+} e^{-} \rightarrow \Upsilon(1 S) K^{+} K^{-}\right) / \sigma\left(e^{+} e^{-} \rightarrow\right.$ $\left.\Upsilon(1 S) K^{0} \bar{K}^{0}\right)=1 / 4$. These features provide crucial tests of the tetraquark framework and can be searched for in the currently available and forthcoming data from the $B$ factories.
\end{abstract}

PACS numbers: 14.40.Rt, 14.40.Pq, 13.66.Bc

The anomalously large production cross sections for $e^{+} e^{-} \rightarrow \Upsilon(1 S) \pi^{+} \pi^{-}, \Upsilon(2 S) \pi^{+} \pi^{-}$and $e^{+} e^{-} \rightarrow$ $\Upsilon(3 S) \pi^{+} \pi^{-}$measured between $\sqrt{s}=10.83 \mathrm{GeV}$ and $11.02 \mathrm{GeV}$ by the Belle Collaboration [1, 2] at KEK do not agree well with the lineshape and production rates for the conventional $b \bar{b}$ state $\Upsilon(10860)$ (also called $\Upsilon(5 S)$ ). A fit to the measured production cross sections using a Breit-Wigner resonance shape yielded a peak mass of $\left[10888.4_{-2.6}^{+2.7}\right.$ (stat) \pm 1.2 (syst) $] \mathrm{MeV}$ and a width of $\left[30.7_{-7.0}^{+8.3}\right.$ (stat) \pm 3.1 (syst) $] \mathrm{MeV}$ for the observed state, henceforth called $Y_{b}(10890)$ [2]. More data are required to understand the resonance structure in this region. In [3], a dynamical model was developed to explain the Belle data for the final states $\Upsilon(1 S) \pi^{+} \pi^{-}$and $\Upsilon(2 S) \pi^{+} \pi^{-}$in terms of the production and decays of the states $Y_{[b, l / h]}$, which are linear superposition of the $J^{P C}=1^{--}$hidden $b \bar{b}$ tetraquark states $Y_{[b u]} \equiv[b u][\bar{b} \bar{u}]$ and $Y_{[b d]} \equiv[b d][\bar{b} \bar{d}]$. The mass difference, estimated as $M\left(Y_{[b, h]}\right)-M\left(Y_{[b, l]}\right)=(5.6 \pm 2.8) \mathrm{MeV}[4,[5]$, was ignored and the mass-degenerate states $Y_{[b, l]}$ and $Y_{[b, h]}$ were identified with the $Y_{b}(10890)$. This model described the distributions in the dipion invariant mass and the helicity angle measured by Belle [1] well and offered an explanation of the rates in terms of the Zweig-allowed transitions $Y_{[b, l / h]} \rightarrow(\Upsilon(1 S), \Upsilon(2 S), \Upsilon(3 S)) \pi^{+} \pi^{-}$. In the case of $X(3872)$, the phase space for the decay $X(3872) \rightarrow D D^{*}$ is highly constrained, yielding a very small binding energy: $\mathcal{E}_{0} \simeq M_{X}-M_{D}-M_{D^{*}}=-0.25 \pm 0.40 \mathrm{MeV}$. This led to suggestions that $X(3872)$ could be a hadron molecule. In the case of $Y_{b}(10890)$, the phase space is large enough to allow the decays $Y_{b}(10890) \rightarrow B^{(*)} \bar{B}^{(*)}$, and consequently the decay width is large, $\Gamma_{Y_{b}} \simeq 30$ $\mathrm{MeV}$, which is of the same order as the total width of the $\Upsilon(5 S)$. Hence, $Y_{b}(10890)$, in all likelihood, is not a hadron molecule. While credible, the interpretation of $Y_{b}(10890)$ in terms of the $b \bar{b}$ tetraquark states requires further experimental scrutiny. It is the aim of this Letter to provide some definitive tests to confirm or rule out the tetraquark interpretation of the Belle data.

To that end, we further develop the tetraquark formalism for the processes

$$
e^{+}+e^{-} \rightarrow Y_{b}(q) \rightarrow \Upsilon(1 S)(p)+P\left(k_{1}\right)+P^{\prime}\left(k_{2}\right),
$$

where $P P^{\prime}$ stands for the pseudoscalar-meson pairs $\pi^{+} \pi^{-}, K^{+} K^{-}$and $\eta \pi^{0}$, and $q, p, k_{1}$ and $k_{2}$ are the momenta of $Y_{b}, \Upsilon(1 S), P$ and $P^{\prime}$, respectively. We neglect other background processes, based on prior data on the dipionic transitions involving higher $\Upsilon(n S)$ to lower $\Upsilon(m S)(m<n)$ states [6]. Following [7], the low mass scalar $0^{++}$hadrons $\sigma$ or $f_{0}(600), f_{0}(980)$ and $a_{0}^{0}(980)$ (the upper index indicates the $I_{3}=0$ component of the iso-triplet $\left.a_{0}\right)$, which enter as intermediate states in the processes $Y_{b} \rightarrow \Upsilon(1 S)+\left[\sigma, f_{0}(980), \cdots\right] \rightarrow \Upsilon(1 S) P P^{\prime}$, are assumed to be tetraquark states. These intermediate $J^{\mathrm{PC}}=0^{++}$states together with the $J^{\mathrm{PC}}=2^{++}$ state $f_{2}(1270)$ provide the dominant resonating part of the amplitudes for the processes considered in this work. These resonances are labeled $\sigma, f_{0}, a_{0}^{0}$ and $f_{2}$ henceforth. We determine the coupling constants involving these light tetraquark states and the mesons $P P^{\prime}$ from the known decays from the PDG [6] and data from the E791 [8], the BES [9], the Crystal Barrel (CB) [10] and the KLOE Collaborations [11, 12], adopting the Flatté model [13] for the $\sigma, f_{0}$ and $a_{0}^{0}$ couplings to take into account threshold effects. The non-resonating continuum contributions are parameterized in terms of two a priori unknown constants [14]. With this formalism, we analyze the invariant-mass $M_{P P^{\prime}}$ and the $\cos \theta$ spectra, where $M_{P P^{\prime}}=\left(k_{1}+k_{2}\right)^{2}$ and $\theta$ is the angle between the momenta of $Y_{b}$ and $P$ in the $P P^{\prime}$ rest frame.

The theoretical framework described here provides good fits of the Belle data on the invariant dipion mass spectrum and $\cos \theta$ distribution in the process $e^{+} e^{-} \rightarrow$ $\Upsilon(1 S) \pi^{+} \pi^{-}$and the ratio $\sigma_{\Upsilon(1 S) K^{+} K^{-}} / \sigma_{\Upsilon(1 S) \pi^{+} \pi^{-}}$, with $\sigma_{\Upsilon(1 S) P P^{\prime}}$ being the cross section $\sigma\left(e^{+} e^{-} \rightarrow\right.$ $\left.\Upsilon(1 S) P P^{\prime}\right)$. We present the invariant mass distribu- 
tions for the $K^{+} K^{-}$and $\eta \pi^{0}$ mesons in the processes $e^{+} e^{-} \rightarrow \Upsilon(1 S)\left(K^{+} K^{-}, \eta \pi^{0}\right)$, which are dominated by the respective $J^{P C}=0^{++}$resonances. The resulting correlations among $\sigma_{\Upsilon(1 S) \pi^{+} \pi^{-}}, \sigma_{\Upsilon(1 S) K^{+} K^{-}}$and $\sigma_{\Upsilon(1 S) \eta \pi^{0}}$ are worked out. Constraining these correlations from the existing data on the first two processes, we predict $\sigma_{\Upsilon(1 S) \eta \pi^{0}} / \sigma_{\Upsilon(1 S) \pi^{+} \pi^{-}}$. We also predict $\sigma_{\Upsilon(1 S) K^{+} K^{-}} / \sigma_{\Upsilon(1 S) K^{0} \bar{K}^{0}}=1 / 4$, reflecting the ratio $Q_{[b u]}^{2} / Q_{[b d]}^{2}$ with $Q_{[b u]}=1 / 3$ and $Q_{[b d]}=-2 / 3$ being the effective electric charges for the constituent diquarks of $Y_{[b u]}$ and $Y_{[b d]}$, respectively.

We start by defining the tetraquark states in the isospin basis, with the two isospin components $Y_{b}^{0} \equiv$ $\left(Y_{[b u]}+Y_{[b d]}\right) / \sqrt{2}$ and $Y_{b}^{1} \equiv\left(Y_{[b u]}-Y_{[b d]}\right) / \sqrt{2}$ for isospin $I=0$ and $I=1$, respectively. The two mass eigenstates $Y_{[b, l]}$ and $Y_{[b, h]}$ are identified with $Y_{[b u]}$ and $Y_{[b d]}$, as the mixings between them is small. We ignore the mass difference and also the isospin breaking effects except for the production processes $e^{+} e^{-} \rightarrow Y_{b}^{I}$ hereafter.

We calculate the decay amplitude as a sum of the BreitWigner resonances and non-resonating continuum contributions, with the latter adopted from [14]. The differential cross section is then written as

$$
\begin{aligned}
& \frac{d^{2} \sigma_{\Upsilon(1 S) P P^{\prime}}}{d M_{P P^{\prime}} d \cos \theta}=\frac{\lambda^{1 / 2}\left(s, m_{\Upsilon}^{2}, M_{P P^{\prime}}^{2}\right) \lambda^{1 / 2}\left(M_{P P^{\prime}}^{2}, m_{P}^{2}, m_{P^{\prime}}^{2}\right)}{384 \pi^{3} s M_{P P^{\prime}}\left[\left(s-m_{Y_{b}}^{2}\right)^{2}+m_{Y_{b}}^{2} \Gamma_{Y_{b}}^{2}\right]} \\
& \times\left\{\left(1+\frac{(q \cdot p)^{2}}{2 s m_{\Upsilon}^{2}}\right)|\mathcal{S}|^{2}\right. \\
& \quad+2 \operatorname{Re}\left[\mathcal{S}^{*}\left(\mathcal{D}^{\prime}+\frac{(q \cdot p)^{2}}{2 s m_{\Upsilon}^{2}} \mathcal{D}^{\prime \prime}\right)\right]\left(\cos ^{2} \theta-\frac{1}{3}\right) \\
& \quad+|\mathcal{D}|^{2} \sin ^{2} \theta\left[\sin ^{2} \theta+2\left(\frac{\left(q^{0}\right)^{2}}{s}+\frac{\left(p^{0}\right)^{2}}{m_{\Upsilon}^{2}}\right) \cos ^{2} \theta\right] \\
& \left.\quad+\left(\left|\mathcal{D}^{\prime}\right|^{2}+\frac{(q \cdot p)^{2}}{2 s m_{\Upsilon}^{2}}\left|\mathcal{D}^{\prime \prime}\right|^{2}\right)\left(\cos ^{2} \theta-\frac{1}{3}\right)^{2}\right\}
\end{aligned}
$$

where $\lambda(x, y, z) \equiv(x-y-z)^{2}-4 y z, q^{0}$ and $p^{0}$ are the energies of the $Y_{b}$ and $\Upsilon(1 S)$ in the $P P^{\prime}$ rest frame, respectively, $\Gamma_{Y_{b}}$ is the decay width of $Y_{b}$, and $m_{Y_{b}}, m_{\Upsilon}$, $m_{P}$ and $m_{P^{\prime}}$ are the masses of $Y_{b}, \Upsilon(1 S), P$ and $P^{\prime}$, respectively. We take $m_{Y_{b}}=10.89 \mathrm{GeV}$ and $\Gamma_{Y_{b}}=30 \mathrm{MeV}$ in the numerical analysis below. A detailed derivation of the above formula will be presented in [15].

Each $P P^{\prime}$ channel receives specified contributions depending on the isospin of $P P^{\prime}$ and the kinematically allowed region for the invariant mass $M_{P P^{\prime}} \in\left[m_{P}+\right.$ $\left.m_{P^{\prime}}, \sqrt{s}-m_{\Upsilon(1 S)}\right]$. The S-wave amplitude for the $P P^{\prime}$ system, $\mathcal{S}$, and the D-wave amplitudes, $\mathcal{D}, \mathcal{D}^{\prime}$ and $\mathcal{D}^{\prime \prime}$, are the sums over possible isospin states

$$
\mathcal{M}=\sum_{I} \mathcal{M}_{I} \quad \text { for } \quad \mathcal{M}=\mathcal{S}, \mathcal{D}, \mathcal{D}^{\prime}, \mathcal{D}^{\prime \prime}
$$

where $I=0$ for $\pi^{+} \pi^{-}, I=0,1$ for $K^{+} K^{-}$, and $I=1$ for $\eta \pi^{0}$, since the $\Upsilon(1 S)$ is an isospin 0 state, and the following resonances contribute to each process:

$$
\begin{array}{lll}
\sigma, f_{0} \text { and } f_{2} & \text { for } & \pi^{+} \pi^{-}, \\
f_{0}, a_{0}^{0} \text { and } f_{2} & \text { for } & K^{+} K^{-}, \\
a_{0}^{0} & \text { for } & \eta \pi^{0} .
\end{array}
$$

The $I=0$ amplitudes are given by the combinations of the resonance amplitudes, $\mathcal{M}_{0}^{S}$ and $\mathcal{M}_{0}^{f_{2}}$, and the nonresonating continuum amplitudes, $\mathcal{M}_{0}^{1 C}$ and $\mathcal{M}_{0}^{2 C}$ :

$$
\begin{aligned}
& \mathcal{S}_{0}=\mathcal{M}_{0}^{1 C}+\left(k_{1} \cdot k_{2}\right) \sum_{S} \mathcal{M}_{0}^{S}, \quad \mathcal{D}_{0}=|\boldsymbol{k}|^{2} \mathcal{M}_{0}^{f_{2}}, \\
& \mathcal{D}_{0}^{\prime}=\mathcal{M}_{0}^{2 C}-\mathcal{D}_{0}, \quad \mathcal{D}_{0}^{\prime \prime}=\mathcal{M}_{0}^{2 C}+\frac{2 q^{0} p^{0}}{(q \cdot p)} \mathcal{D}_{0},
\end{aligned}
$$

where $S$ runs over possible $I=0$ scalar resonances in Eq. (4), and $|\boldsymbol{k}|$ is the magnitude of the three momentum of $P^{(\prime)}$ in the $P P^{\prime}$ rest frame. Similarly, the $I=1$ amplitudes are given by

$$
\begin{aligned}
& \mathcal{S}_{1}=\frac{g_{e^{+} e^{-} Y_{b}^{1}}}{g_{e^{+} e^{-} Y_{b}^{0}}}\left[\mathcal{M}_{1}^{1 C}+\left(k_{1} \cdot k_{2}\right) \mathcal{M}_{1}^{a_{0}^{0}}\right], \\
& \mathcal{D}_{1}=0, \quad \mathcal{D}_{1}^{\prime}=\mathcal{D}_{1}^{\prime \prime}=\frac{g_{e^{+} e^{-} Y_{b}^{1}}}{g_{e^{+} e^{-} Y_{b}^{0}}} \mathcal{M}_{1}^{2 C},
\end{aligned}
$$

where the dimensionless couplings $g_{e^{+} e^{-} Y_{b}^{I}}$ are defined through the Lagrangian $\mathcal{L}=\sum_{I=0,1} g_{e^{+} e^{-} Y_{b}^{I}} Y_{b \mu}^{I}\left(\bar{e} \gamma^{\mu} e\right)$, and the ratio is given by $g_{e^{+} e^{-} Y_{b}^{1}} / g_{e^{+} e^{-} Y_{b}^{0}}=\left(Q_{[b u]}-\right.$ $\left.Q_{[b d]}\right) /\left(Q_{[b u]}+Q_{[b d]}\right)=-3$.

To calculate the production cross sections, we derive the corresponding Van Royen-Weisskopf formula for the leptonic decay widths of the tetraquark states made up of point-like diquarks:

$$
\Gamma\left(Y_{[b u / b d]} \rightarrow e^{+} e^{-}\right)=\frac{24 \alpha^{2}\left|Q_{[b u / b d]}\right|^{2}}{m_{Y_{b}}^{4}} \kappa^{2}\left|R_{11}^{(1)}(0)\right|^{2},
$$

where $\alpha$ is the fine-structure constant, the parameter $\kappa$ takes into account differing sizes of the tetraquarks compared to the standard bottomonia as well as the hadronic size of the diquarks, with $\kappa<1$ anticipated, and $\left|R_{11}^{(1)}(0)\right|^{2}=2.067 \mathrm{GeV}^{5}[16]$ is the square of the derivative of the radial wave function for $\chi_{b}(1 P)$ taken at the origin. Hence, the leptonic widths of the tetraquark states are estimated as

$$
\Gamma\left(Y_{[b d]} \rightarrow e^{+} e^{-}\right)=4 \Gamma\left(Y_{[b u]} \rightarrow e^{+} e^{-}\right) \approx 83 \kappa^{2} \mathrm{eV},
$$

which are substantially smaller than the leptonic width of the $\Upsilon(5 S)$ [6]. Combining the knowledge of the production process and the measured cross section for $e^{+} e^{-} \rightarrow$ $\Upsilon(1 S) \pi^{+} \pi^{-}$, we estimate $\Gamma\left(Y_{[b u / b d]} \rightarrow \Upsilon(1 S) \pi^{+} \pi^{-}\right)$to be $O(1) \mathrm{MeV}$.

The continuum amplitudes in Eq. (5) are written in terms of the two form factors $A$ and $B[14,15]$ as

$$
\begin{aligned}
& \mathcal{M}_{0}^{1 C}=\frac{2 A^{\prime}}{f_{P} f_{P^{\prime}}}\left(k_{1} \cdot k_{2}\right)+\frac{B^{\prime}}{f_{P} f_{P^{\prime}}} \frac{3\left(q^{0}\right)^{2} k_{1}^{0} k_{2}^{0}-|\boldsymbol{q}|^{2}|\boldsymbol{k}|^{2}}{3 s}, \\
& \mathcal{M}_{0}^{2 C}=-\frac{B^{\prime}}{f_{P} f_{P^{\prime}}} \frac{|\boldsymbol{q}|^{2}|\boldsymbol{k}|^{2}}{s}
\end{aligned}
$$


where the primed quantities here, and later, are rescaled as $A^{\prime}=A g_{e^{+} e^{-} Y_{b}^{0}}$ and $B^{\prime}=B g_{e^{+} e^{-} Y_{b}^{0}}, f_{P^{(\prime)}}$ is the decay constant of $P^{(\prime)}$, and $|\boldsymbol{q}|, k_{1}^{0}$ and $k_{2}^{0}$ are the magnitude of the three momentum of $Y_{b}$ and the energies of $P$ and $P^{\prime}$ in the $P P^{\prime}$ rest frame, respectively. Using SU(3) symmetry for the form factors $A$ and $B$ in Eq. (9), but not for the pseudoscalar meson masses and coupling constants, we assume the relations $\mathcal{M}_{0}^{1 C, 2 C}\left(\Upsilon(1 S) K^{+} K^{-}\right)=$ $(\sqrt{3} / 2) \mathcal{M}_{0}^{1 C, 2 C}\left(\Upsilon(1 S) \pi^{+} \pi^{-}\right), \mathcal{M}_{1}^{1 C, 2 C}\left(\Upsilon(1 S) K^{+} K^{-}\right)=$ $\mathcal{M}_{0}^{1 C, 2 C}\left(\Upsilon(1 S) K^{+} K^{-}\right)$and $\mathcal{M}_{1}^{1 C, 2 C}\left(\Upsilon(1 S) \eta \pi^{0}\right)=$ $\sqrt{2} \mathcal{M}_{1}^{1 C, 2 C}\left(\Upsilon(1 S) K^{+} K^{-}\right)$.

The resonant contributions are expressed by the BreitWigner formula:

$$
\mathcal{M}_{I}^{R}=\frac{g_{R P P^{\prime}} g_{Y_{b}^{I} \Upsilon(1 S) R} g_{e^{+} e^{-} Y_{b}^{0}}}{M_{P P^{\prime}}^{2}-m_{R}^{2}+i m_{R} \Gamma_{R}} e^{i \varphi_{R}},
$$

where $I=0$ for $R=\sigma, f_{0}$ and $f_{2}$, and $I=1$ for $R=a_{0}^{0}$. The couplings for the scalar resonances $S$ are defined through the Lagrangian $\mathcal{L}=g_{S P P^{\prime}}\left(\partial_{\mu} P\right)\left(\partial^{\mu} P^{\prime}\right) S+$ $g_{Y_{b} \Upsilon(1 S) S} Y_{b \mu} \Upsilon^{\mu} S$, while those for the $f_{2}$ are defined via $\mathcal{L}=2 g_{f_{2} P P^{\prime}}\left(\partial_{\mu} P\right)\left(\partial_{\nu} P^{\prime}\right) f_{2}^{\mu \nu}+g_{Y_{b}} \Upsilon(1 S) f_{2} Y_{b \mu} \Upsilon_{\nu} f_{2}^{\mu \nu}$. The couplings $g_{R P P^{\prime}}$ and $g_{Y_{b}^{I}} \Upsilon(1 S) R$ have mass dimensions -1 and 1 , respectively. For the $\sigma, f_{0}$ and $a_{0}^{0}$, we adopt the Flatté model [13]

$$
\begin{aligned}
& m_{\sigma} \Gamma_{\sigma}=f_{\sigma \pi \pi}^{2} \rho_{\pi \pi}, \quad m_{f_{0}} \Gamma_{f_{0}}=f_{f_{0} \pi \pi}^{2} \rho_{\pi \pi}+f_{f_{0} K \bar{K}}^{2} \rho_{K \bar{K}} \\
& m_{a_{0}^{0}} \Gamma_{a_{0}^{0}}=f_{a_{0}^{0} \eta \pi}^{2} \rho_{\eta \pi}+f_{a_{0}^{0} K \bar{K}}^{2} \rho_{K \bar{K}}
\end{aligned}
$$

with the phase space factor $\rho_{a b}=\left[\left(1-\left(m_{a}-\right.\right.\right.$ $\left.\left.\left.m_{b}\right)^{2} / M_{P P^{\prime}}^{2}\right)\left(1-\left(m_{a}+m_{b}\right)^{2} / M_{P P^{\prime}}^{2}\right)\right]^{1 / 2}$, where the Flatté couplings $f_{S P P^{\prime}}$ are related to the vertex couplings $g_{S P P^{\prime}}$ entering in Eq. (10) via

$$
g_{S P P^{\prime}}\left(k_{1} \cdot k_{2}\right)=4 \sqrt{\pi} f_{S P P^{\prime}} .
$$

The couplings in Eq. (12) are defined for an exclusive final state. Summing over final states, e.g., $f_{f_{0} \pi \pi}^{2}=$ $f_{f_{0} \pi^{+} \pi^{-}}^{2}+f_{f_{0} \pi^{0} \pi^{0}}^{2}$, we obtain the isospin relations $f_{S \pi \pi}=$ $\sqrt{3 / 2} f_{S \pi^{+} \pi^{-}}, f_{S K \bar{K}}=\sqrt{2} f_{S K^{+} K^{-}}$and $f_{S \eta \pi}=f_{S \eta \pi^{0}}$. For the $\sigma$ meson, we extract the coupling $g_{\sigma \pi^{+} \pi^{-}}$from the E791 data [8]: $g_{\sigma \pi^{+} \pi^{-}}=26.7 \mathrm{GeV}^{-1}$ with $m_{\sigma}=478$ $\mathrm{MeV}$, yielding the Flatté coupling $f_{\sigma \pi \pi}=437 \mathrm{MeV}$. For the $f_{0}$ and $a_{0}^{0}$ mesons, we adopt the masses and the Flatté couplings measured by the BES [9] and CB [10] Collaborations (the corresponding couplings from KLOE [11, 12] are shown in the parentheses):

$$
\begin{aligned}
& m_{f_{0}}=965(984), f_{f_{0} \pi \pi}=406(349), f_{f_{0} K \bar{K}}=833(869) \\
& m_{a_{0}^{0}}=982(983), f_{a_{0}^{0} \eta \pi}=324(398), f_{a_{0}^{0} K \bar{K}}=329(429)
\end{aligned}
$$

in units of MeV. Furthermore, we extract the couplings for the $f_{2}$ meson from $\Gamma\left(f_{2} \rightarrow P P^{\prime}\right)=g_{f_{2} P P^{\prime}}^{2} m_{f_{2}}^{3}(1-$ $\left.4 m_{P}^{2} / m_{f_{2}}^{2}\right)^{5 / 2} /(480 \pi)$ for $m_{P}=m_{P^{\prime}}$, where the data for $\Gamma\left(f_{2} \rightarrow \pi \pi\right)=(3 / 2) \Gamma\left(f_{2} \rightarrow \pi^{+} \pi^{-}\right)$and $\Gamma\left(f_{2} \rightarrow K \bar{K}\right)=$ $2 \Gamma\left(f_{2} \rightarrow K^{+} K^{-}\right)$, and $m_{f_{2}}=1275 \mathrm{MeV}$ are taken from
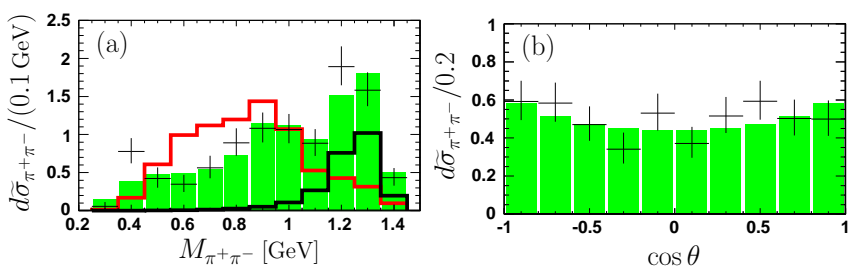

FIG. 1. Fit results (a) of the $M_{\pi^{+} \pi^{-}}$distribution and (b) of the $\cos \theta$ distribution for $e^{+} e^{-} \rightarrow Y_{b} \rightarrow \Upsilon(1 S) \pi^{+} \pi^{-}$, normalized by the measured cross section. The histograms (green bars) represent the fit results, while the crosses are the Belle data [1]. The resonant contributions from the $\sigma$ and $f_{0}(980)$ (left red curve) and the $f_{2}(1270)$ (right black curve) are also indicated in (a).

TABLE I. Best fit parameters, yielding $\chi^{2} /$ d.o.f. $=21.5 / 15$, where $A^{\prime}$ and $B^{\prime}$ are dimensionless, $g_{Y_{b}^{0} \Upsilon(1 S) f_{0}}^{\prime}$ and $g_{Y_{b}^{0} \Upsilon(1 S) f_{2}}^{\prime}$ are given in units of $\mathrm{MeV}$, and the angles are in units of rad.

\begin{tabular}{ccccccc}
\hline \hline$A^{\prime}$ & $B^{\prime}$ & $g_{Y_{b}^{0} \Upsilon(1 S) f_{0}}^{\prime}$ & $g_{Y_{b}^{0} \Upsilon(1 S) f_{2}}^{\prime}$ & $\varphi_{\sigma}$ & $\varphi_{f_{0}}$ & $\varphi_{f_{2}}$ \\
\hline 0.000079 & -0.00020 & 0.318 & 0.439 & 0.36 & -2.76 & -0.46 \\
\hline \hline
\end{tabular}

PDG [6]. The other inputs for the pseudo-scalar mesons and the $\Upsilon(1 S)$ are also taken from PDG.

Having detailed our dynamical model, we now perform a simultaneous fit to the binned $\Upsilon(1 S) \pi^{+} \pi^{-}$ data for the $M_{\pi^{+} \pi^{-}}$and $\cos \theta$ distributions measured by Belle at $\sqrt{s}=10.87 \mathrm{GeV}$ [1], normalizing them by the measured cross section: $d \widetilde{\sigma}_{\pi^{+} \pi^{-}} / d M_{\pi^{+} \pi^{-}}$and $d \widetilde{\sigma}_{\pi^{+} \pi^{-}} / d \cos \theta$, where $\widetilde{\sigma}_{\pi^{+} \pi^{-}} \equiv \sigma_{\Upsilon(1 S) \pi^{+} \pi^{-}} / \sigma_{\Upsilon(1 S) \pi^{+} \pi^{-}}^{\text {Belle }}$ with $\sigma_{\Upsilon(1 S) \pi^{+} \pi^{-}}^{\text {Belle }}=1.61 \pm 0.16 \mathrm{pb}[1]$. With $\mathrm{SU}(3)$ symmetry for the $Y_{b}^{0} \Upsilon(1 S) R$ couplings entering in Eq. (10), i.e., setting $g_{Y_{b}^{0} \Upsilon(1 S) \sigma}=g_{Y_{b}^{0} \Upsilon(1 S) f_{0}}$, we have 7 free parameters:

$$
A^{\prime}, B^{\prime}, g_{Y_{b}^{0} \Upsilon(1 S) f_{0}}^{\prime}, g_{Y_{b}^{0} \Upsilon(1 S) f_{2}}^{\prime}, \varphi_{\sigma}, \varphi_{f_{0}}, \varphi_{f_{2}} .
$$

Hence the number of degrees of freedom (d.o.f.) in the fit is $(12+10)-7=15$. To make predictions for the $\Upsilon(1 S) K^{+} K^{-}$and $\Upsilon(1 S) \eta \pi^{0}$ modes, we assume $\mathrm{SU}(3)$ for the couplings and the phases, i.e., $g_{Y_{b}^{0} \Upsilon(1 S) f_{0}}=$ $g_{Y_{b}^{1} \Upsilon(1 S) a_{0}^{0}}$ and $\varphi_{f_{0}}=\varphi_{a_{0}^{0}}$. We also assume that there is no phase difference between the two continuum amplitudes $\mathcal{M}_{0}^{1 C, 2 C}$ and $\mathcal{M}_{1}^{1 C, 2 C}$.

With these inputs, we have performed a large number of fits (typically $O(5000)$ ) of the Belle data with the tetraquark theory predictions. The resultant best fit is fairly good, with $\chi^{2} /$ d.o.f. $=21.5 / 15$ for the BES and CB input in Eq. (13), which corresponds to a $p$-value of 0.12 . The corresponding best fit using the KLOE data is very similar, having a $\chi^{2} /$ d.o.f. $=21.9 / 15$, yielding a $p$-value of 0.11. The best fit using the BES and CB data is presented in Fig. 1 and the corresponding fit values of the parameters are listed in Table [. Further details about 

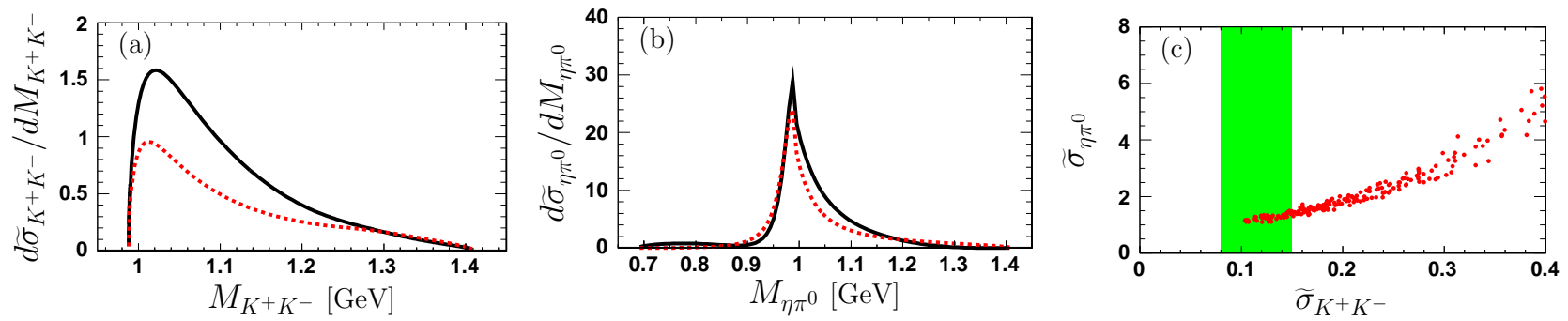

FIG. 2. Predictions (a) of the $M_{K^{+} K^{-}}$distribution for $e^{+} e^{-} \rightarrow Y_{b} \rightarrow \Upsilon(1 S) K^{+} K^{-}$, (b) of the $M_{\eta \pi^{0}}$ distribution for $e^{+} e^{-} \rightarrow$ $Y_{b} \rightarrow \Upsilon(1 S) \eta \pi^{0}$ and (c) of the correlation between the cross sections of $\Upsilon(1 S) K^{+} K^{-}$and $\Upsilon(1 S) \eta \pi^{0}$, normalized by the measured cross section for the $\Upsilon(1 S) \pi^{+} \pi^{-}$mode. In (a) and (b), the dotted (solid) curves show the dimeson invariant mass spectra from the resonant (total) contribution. In (c), the red dots represent predictions from our fit solutions satisfying $\chi^{2} /$ d.o.f. $<1.6$. The shaded (green) band shows the current Belle measurement $\widetilde{\sigma}_{K^{+} K^{-}}=0.11_{-0.03}^{+0.04}[1]$.

the correlations among the parameters and the cross sections will be presented in a forthcoming paper [15]. Concluding the discussion of the final state $\Upsilon(1 S) \pi^{+} \pi^{-}$, we note that the resonance contribution represented by the left red curve (S-wave from $\sigma$ and $f_{0}$ ) and the right black curve (D-wave from $f_{2}$ ) in Fig. 1(a) dominate the $M_{\pi^{+} \pi^{-}}$ spectrum, supporting our dynamical model in the decay $Y_{b} \rightarrow \Upsilon(1 S) \pi^{+} \pi^{-}$. Sufficient data may provide enough statistics to undertake an analysis in the end-region of $M_{\pi^{+} \pi^{-}}$to probe the angular distribution of $f_{2} \rightarrow \pi^{+} \pi^{-}$.

The normalized $M_{K^{+} K^{-}}$and $M_{\eta \pi^{0}}$ distributions, calculated with the best-fit parameters in Table@ are shown in Fig. 2 (a) and Fig. 2 (b), respectively. In these figures, the dotted (solid) curves show the dimeson invariant mass spectra from the resonant (total) contribution. Since these spectra are dominated by the scalars $f_{0}+a_{0}^{0}$ and $a_{0}^{0}$, respectively, there is a strong correlation between the two cross sections. This is shown in Fig. 2 (c), where we have plotted the normalized cross sections $\widetilde{\sigma}_{K^{+} K^{-}}$ and $\widetilde{\sigma}_{\eta \pi^{0}}$ resulting from our fits (dotted points) which all satisfy $\chi^{2} /$ d.o.f. $<1.6$. The current Belle measurement $\widetilde{\sigma}_{K^{+} K^{-}}=0.11_{-0.03}^{+0.04}[1]$ is shown as a shaded (green) band on this figure. Our model is in agreement with the Belle measurement, though there is a tendency in the fits to yield larger value for $\widetilde{\sigma}_{K^{+} K^{-}}$. Our predictions will be further tested as and when the cross section $\widetilde{\sigma}_{\eta \pi^{0}}$ is measured. Noticing that we have neglected the SU(3)breaking effects, we predict $1.0 \lesssim \widetilde{\sigma}_{\eta \pi^{0}} \lesssim 2.0$.

Finally, we note that the states $\Upsilon(1 S) K^{+} K^{-}$and $\Upsilon(1 S) K^{0} \bar{K}^{0}$ are produced by the underlying mechanism $e^{+} e^{-} \rightarrow Y_{[b u]} \rightarrow \Upsilon(1 S) K^{+} K^{-}$and $e^{+} e^{-} \rightarrow Y_{[b d]} \rightarrow$ $\Upsilon(1 S) K^{0} \bar{K}^{0}$. Hence, a firm prediction is

$$
\frac{\sigma_{\Upsilon(1 S) K^{+} K^{-}}}{\sigma_{\Upsilon(1 S) K^{0} \bar{K}^{0}}}=\frac{Q_{[b u]}^{2}}{Q_{[b d]}^{2}}=\frac{1}{4} .
$$

This relation is valid under the assumption that the diquarks are point-like. In terms of the mass eigenstates, we predict $\sigma_{\Upsilon(1 S) K^{+} K^{-}}=\sigma_{\Upsilon(1 S) K_{S} K_{S}}$.
The distributions, cross sections, and correlations presented here are crucial tests of the underlying tetraquark hypothesis in the $b \bar{b}$ sector and go well beyond what has been proposed in the literature to understand the nature of the $Y_{b}(10890)$ state 3 . They will be scrutinized soon in the existing and the forthcoming Belle data to which we look forward.

* ahmed.ali@desy.de

† christian.hambrock@desy.de

‡ satoshi.mishima@desy.de

[1] K. F. Chen et al. [Belle Collaboration], Phys. Rev. Lett. 100, 112001 (2008).

[2] I. Adachi et al. [Belle Collaboration], arXiv:0808.2445 [hep-ex].

[3] A. Ali, C. Hambrock, and M. J. Aslam, Phys. Rev. Lett. 104, 162001 (2010).

[4] A. Ali, C. Hambrock, I. Ahmed, and M. J. Aslam, Phys. Lett. B 684, 28 (2010).

[5] L. Maiani, F. Piccinini, A. D. Polosa, and V. Riquer, Phys. Rev. D 71, 014028 (2005).

[6] C. Amsler et al. [Particle Data Group], Phys. Lett. B 667, 1 (2008).

[7] G. 't Hooft et al., Phys. Lett. B 662, 424 (2008); A. H. Fariborz, R. Jora, and J. Schechter, Phys. Rev. D 77, 094004 (2008).

[8] E. M. Aitala et al. [E791 Collaboration], Phys. Rev. Lett. 86, 770 (2001).

[9] M. Ablikim et al. [BES Collaboration], Phys. Lett. B 607, 243 (2005).

[10] A. Abele et al. [Crystal Barrel Collaboration], Phys. Rev. D 57, 3860 (1998).

[11] F. Ambrosino et al. [KLOE Collaboration], Nucl. Phys. Proc. Suppl. 186, 290 (2009).

[12] F. Ambrosino et al. [KLOE Collaboration], Phys. Lett. B 681, 5 (2009).

[13] S. M. Flatte, Phys. Lett. B 63, 224 (1976).

[14] L. S. Brown and R. N. Cahn, Phys. Rev. Lett. 35, 1 (1975).

[15] A. Ali, C. Hambrock, and S. Mishima, to appear.

[16] E. J. Eichten and C. Quigg, Phys. Rev. D 52, 1726 (1995). 